\title{
The Cryptocurrency Uncertainty Index
}

\author{
Brian M. Lucey ${ }^{a, b, c, d}$, Samuel A. Vigne ${ }^{a}$, Larisa Yarovaya ${ }^{e},{\text { Yizhi } \mathrm{Wang}^{a}}^{a}$ \\ ${ }^{a}$ Trinity Business School, Trinity College Dublin, Dublin 2, Ireland \\ ${ }^{b}$ University of Sydney Business School, University of Sydney, Sydney, New South Wales, Australia \\ ${ }^{c}$ Distinguished Research Fellow, Institute of Business Research, University of Economics Ho Chi Minh City, 59C \\ Nguyen Dinh Chieu, Ward 6, District 3, Ho Chi Minh City, Vietnam \\ ${ }^{d}$ Institute for Industrial Economics, Jiangxi University of Economics and Finance, 169, East Shuanggang Road, \\ Xialuo, Changbei District 330013 Nanchang, Jiangxi, China \\ e Center for Digital Finance, University of Southampton, Highfield Campus, Southampton, UK \\ *Corresponding Author: Brian M. Lucey blucey@tcd.ie; Yizhi Wangwangy27@tcd.ie
}

\begin{abstract}
We have developed and made available a new Cryptocurrency Uncertainty Index (UCRY) based on news coverage. Our UCRY Index captures two types of uncertainty: that of the price of cryptocurrency (UCRY Price) and uncertainty of cryptocurrency policy (UCRY Policy). We show that the constructed index exhibits distinct movements around major events in cryptocurrency space. We suggest that this index captures uncertainty beyond Bitcoin, and can be used for academic, policy, and practice-driven research.
\end{abstract}

Keywords: Cryprocurrencies; Policy and price uncertainty; Uncertainty index; Cryptocurrency Uncertainty Index

JEL Code: C43, C54, D80 


\section{Introduction}

How uncertain are investors about cryptocurrencies, and what drives this? This paper poses a construction and analysis of two indices of policy and price uncertainty for cryptocurrencies in order to address the level of uncertainty that investors experience and the motivating factors of such in regards to cryptocurrencies. Uncertainty is an essential determinant of the volatility of cryptocurrency, in large part due to its association with the future earnings of such. Yet different types of uncertainty may have varying impacts and predictive power on cryptocurrency markets. In addition, it is worth noting the difference between price volatility and price uncertainty. Cryptocurrency price volatility measures the size of variations of cryptocurrency returns, and should be the standard deviation of cryptocurrency logarithmic returns between their daily closing prices. Walther et al. [2019] use 17 different economic and financial indices to predict the volatility of cryptocurrencies. They point out that it is driven by global business and a network of interacting driving factors. The Financial Stress Index and the Chinese Policy Uncertainty Index are useful and impactful predictors for the volatility of cryptocurrencies but are overshadowed by the Global Real Economic Activity Index. Cryptocurrency price uncertainty measures the size of unpredictable disturbances in the price of cryptocurrency. Demir et al. [2018] prove that Economic Policy Uncertainty has a predictive power on Bitcoin returns. Large moves in cryptocurrency uncertainty are less frequent but more persistent than moves in cryptocurrency price volatility.

Akyldirim et al. [2020] showed that during times when investors' fears are elevated, cryptocurrency markets experienced an increase in volatility. The authors used VIX (CBOE-traded) and VSTOXX (DAX-traded) volatility indexes as measures of the United States and European financial market risk respectively. Fang et al. [2020] further analysed the impact of the News-based Implied Volatility index (NVIX) on cryptocurrency returns, providing evidence that NVIX, developed by Manela and Moreira [2017], is a more powerful predictor of the long-term volatility in selected cryptocurrencies than the Global Economic Policy Uncertainty index (GEPU) proposed by Davis [2016]. These results indicate that cryptocurrency market volatility might be more susceptible to price uncertainty and investors' perceptions than to policy uncertainty.

Findings reported by Aysan et al. [2019] demonstrate that the Geopolitical Uncertainty Index (GPR) can predict Bitcoin returns and volatility. Conlon et al. [2020] further compared the impacts of GEPU and GPR indexes on cryptocurrency returns, yet found no substantial safe haven or hedging properties of cryptocurrencies against either uncertainty proxies, apart from a weak ability to hedge against GEPU during a bull market. Their results are consistent with other papers in this area, such as Wu et al. [2019], Al Mamun et al. [2020]. Gozgor et al. [2020] analysed the impact of Trade Policy Uncertainty (TPI) on Bitcoin returns and demonstrated a significant positive correlation between returns and uncertainty variables.

Investment sentiments have been found to be useful for predicting cryptocurrency volatility and returns. Corbet et al. [2020b] constructed a sentiment index based on news stories that followed 
the announcements of four macroeconomic indicators: GDP, unemployment, Consumer Price Index (CPI) and durable goods. The results showed that Bitcoin returns responded differently to news than to stock market returns. Furthermore, it was found that the price cryptocurrency's reaction to news and announcements may vary depending on the type of digital assets. Thus, according to Corbet et al. [2020a], currency-based digital assets are likely more susceptible to the US monetary policy announcements, while applications or protocol-based digital assets are immune to these shocks. Similar differences are found for mineable and non-mineable currencies, meaning that the response to various types of uncertainty of some digital assets would be distinct from that of Bitcoin. Yarovaya and Zięba [2020] further classified cryptocurrencies with respect to multiple qualitative factors, such as geographical location of headquarters, founder's origin, underlying platform, and the consensus algorithm. They explored the differences in patterns of interconnectedness patterns between trading volume and returns across cryptocurrencies from different categories. Benedetti and Nikbakht [2021] identified that specific heterogeneous characteristics of digital tokens affected the cryptocurrency returns. The speculative nature of cryptocurrency markets has implications for market efficiency, portfolio diversification, the contagion effect and financial stability literature, see Corbet et al. [2018] for a systematic review of past papers in this field.

This paper is motivated by three main theoretical arguments discussed in the aforementioned literature. Firstly, our interest lay in exploring the effect of potential clientele in cryptocurrency markets, that is, different groups of investors who are attracted to particular kinds of cryptocurrencies. Secondly, due to their speculative nature, cryptocurrencies are attractive to amateur investors who have the potential to interpret publicly available information differently from large institutional investors. Thus, the impact of uncertainty on cryptocurrency markets will depend on types of uncertainty and the type of digital assets. Thirdly, we considered the importance of determinants of cryptocurrency market volatility analysis. We did so due to the explosivity of cryptocurrencies, which has created a new type of information asymmetry that affects other markets and poses a significant threat to financial stability (e.g., Akyildirim et al. [2020]). Therefore, it is important to develop a measure that can capture uncertainty in cryptocurrency markets.

In addition to the previously addressed indices, a few papers attempted to design new uncertainty indexes, for example, Huang and Luk [2020] introduced a new China EPU index using 10 mainland Chinese newspapers. Additionally, Trimborn and Härdle [2018] introduced the CRIX index to assess the markets volatility of cryptocurrencies. Moreover, there are further cryptocurrency market indexes available, such as the market capitalisation-weighted Bloomberg Galaxy index. However, there is presently no designed index that captures uncertainty of cryptocurrency markets' price and policy.

We introduce a new Cryptocurrency Uncertainty Index (UCRY) that captures two main types of uncertainty, Cryptocurrency Policy Uncertainty (UCRY Policy) and Cryptocurrency Price Uncertainty (UCRY Price). These indices can be used to assess how policy and regulatory debates 
affect cryptocurrency returns and volatility, and how this impact differs from reaction to Bitcoin attention in general. It is important to distinguish between two types of cryptocurrency uncertainty, since it may help to better understand the behaviour of different sets of investors in cryptocurrency markets. While informed investors would be sensitive to changes in policy uncertainty, amateurs may react more strongly to general media attention towards cryptocurrencies and their price fluctuations. Increasing institutional interest in digital assets may also make cryptocurrency markets more susceptible to policy uncertainty over time, which further justifies the importance of the indices introduced in this paper.

Thus, in this research note, we gathered 726.9 million news stories from the LexisNexis database spanning January 2014 to January 2021. We designed the UCRY index, which shows the contributions that the UCRY Policy and the UCRY Price indices have made to historical decomposition of the index around key events in cryptocurrency space. This has been compared with other popular uncertainty measures as well as gold and Bitcoin price uncertainties.

The remainder of this paper is organised as follows, section 2 describes the data and methodology used to construct the indices, while section 3 presents the empirical results, and section 4 concludes and discusses the implications of this study.

\section{Data and methodology}

\subsection{Data collection}

We build our index on the construct found in Baker et al. [2016], using the material found in the LexisNexis Business Database as our corpus of literature. This covers a very wide variety of newspapers and news-wire feeds. Notably, this is unlike most previous measures, which have relied almost solely on major newspapers (see for example Rice [2020]). The rationale for using a greater range of sources, including but not limited to news-wire feeds and media news transcripts, was to acknowledge the "social" aspect of cryptocurrencies. As new phenomena, these currencies have become subject to extensive discussion via not just traditional media, but alternative and social media. See as examples papers such as Phillips and Gorse [2017], Subramaniam and Chakraborty [2020] for discussion of social and general media.

We therefore ran the following queries on LexisNexis business. / (uncertain or uncertainty) and price and atl1(Bitcoin or Ethereum or ripple or litecoin or tether or cryptocurrency or cryptocurrencies) and atl1(regulator or regulators or central bank or government)] was the text search string used to ascertain uncertainty around policy issues, while / (uncertain or uncertainty) and price and atl1(Bitcoin or Ethereum or ripple or litecoin or tether or cryptocurrency or cryptocurrencies) ] was used to gather results on uncertainty more broadly.

In addition, we set the option for Group Duplicate to MODERATE so as to avoid duplicate results as much as possible. The queries were performed for each month from January 2014 to 
January $2021^{1}$.

\subsection{UCRY index construction}

The Cryptocurrency Policy Uncertainty Index is calculated as in Equation 1,

$$
\text { UCRY Policyt }=\left(\frac{N_{1 t}-\mu_{1}}{\sigma_{1}}\right)+100
$$

where UCRY Policy $t$ is the value of the Cryptocurrency Policy Uncertainty Index in the weeks $t$ between December 2013 and February 2021. $N_{1 t}$ is the weekly observed value of news articles on LexisNexis business concerning the uncertainty of cryptocurrency policy, $\mu_{1}$ is the mean of these same articles and $\sigma_{1}$ is the standard deviation of such.

The Cryptocurrency Price Uncertainty Index is calculated as in Equation 2

$$
\text { UCRY } \text { Price }_{t}=\left(\frac{N_{2 t}-\mu_{2}}{\sigma_{2}}\right)+100
$$

where UCRY Price $_{t}$ is the value of the Cryptocurrency Price Uncertainty Index in the weeks $t$ between December 2013 and February 2021. $N_{2 t}$ is the weekly observed value of LexisNexis business news articles concerning the uncertainty of cryptocurrency price news articles, $\mu_{2}$ is the mean of these and $\sigma_{2}$ is the standard deviation of such.

The UCRY Policy Index, the Global EPU index, the Vix, the price of Bitcoin, the USFS index of US financial system stress, the USEPU, the gold, and the UCRY Price Index were select as the system variables, as justified in the Introduction section. Each series was identified and recorded in Table 4. We ordered variables as indicated by Equation 3.

$$
\mathbf{Y}_{t}=\left[\begin{array}{c}
\text { UCRY Policy } \\
\text { GlobalEPU }_{t} \\
\text { Vix }_{t} \\
\text { Bitcoin }_{t} \\
\text { USFS } \\
\text { USEPU } \\
\text { Gold }_{t} \\
\text { UCRY Price }
\end{array}\right]
$$

\footnotetext{
${ }^{1}$ Weekly values can be downloaded from here https://brianmlucey.wordpress.com/2021/03/16/ cryptocurrency-uncertainty-index-dataset/
} 
UCRY Policy Index was ordered first because we believe that GlobalEPU, Vix, Bitcoin, USFS, USEPU and the gold can react contemporaneously to uncertainty shocks. Due to the fact that other indexes are generally react faster than UCRY Price Index, the UCRY Price Index was ordered last.

\subsection{Econometric model}

The main use of Vector Autoregression (VAR) models are forecasting and structural analysis Lütkepohl [2005]. The standard VAR is a reduced form model and is designed for stationary data forms. If economic theory is used to provide the link between forecast errors and fundamental structural shocks, the Structural Vector Autoregression (SVAR) model will be used. The Vector Error Correction Model (VECM) adds error correction features to the VAR. VECM is designed for the non-stationary but co-integrated forms of variables. It is also possible to apply the SVAR technique to VECM with cointegrated variables, named SVECM.

Firstly, a stationarity test was performed on the data, in this case the Augment Dickey-Fuller (ADF) test was applied. Table 1 shows that the $\mathrm{p}$-value of each variable was more significant than 0.05 . These evidences that there are unit roots in all variables and that all variables are nonstationary.

Secondly, if we can further prove the forms of our variables are cointegrated, we can use the SVECM. We therefore applied the Johansen test. The optimal lags calculation results are shown in Table 2. The Akaike information criterion, Hannan - Quinn information criterion, Schwartz information criterion, and prediction error information all suggest four as the optimal lag. From Table 3,r $=0$, tested for the presence of cointegration. Since the tested statistic exceeded the $1 \%$ level significantly $(215.11>177.20)$, we have strong evidence that our variables forms are cointegrated.

Guided by the above analysis, we applied a SVECM with identification based on a Cholesky recursive assumption to a VECM to trace the effects of different economic shocks in a system of variables. Historical decomposition is a tool used for the SVECM analysis, and allows for the gathering of information on the contribution of structural shocks to a system of variables can get from a historical decomposition. In order to explore the quantitative contribution of UCRY Policy and UCRY Price shocks to the dynamics of the system variables mentioned in $Y_{t}$ Equation 3, we will performed historical decomposition.

VECM can be expressed as Equation 4

$$
\Delta y_{t}=\alpha \beta^{\prime} y_{t-1}+\Gamma_{1} \Delta y_{t-1}+\cdots+\Gamma_{p-1} \Delta y_{t-p+1}+\Xi^{+} D_{t}+u_{t},
$$

where $y_{t}$ is a $K \times 1$ dimensional vector of variables observed at time $t$. The decomposed cointegrated models $\alpha \beta^{\prime}$ has reduced rank $r=r k\left(\alpha \beta^{\prime}\right)<K$. Additionally, $\alpha$ is a $K \times r$ matrix containing the 
loading coefficients, $\beta$ is also a $K \times r$ matrix containing the co-integrated vectors. $\Gamma_{j}$ is a $K \times K$ shortrun coefficient matrix with $j=1, \cdots, p-1 . u_{t}$ is a k-dimensional unobservable zero mean vector white noise process, and has covariance matrix $\Sigma_{u} . u_{t}$ also denotes the reduced form disturbance. $D_{t}$ is a vector of deterministic terms, and $\Xi^{+}$is the coefficient matrices corresponded with $D_{t}$.

In this way, structural shocks on the system variables $y_{t}$ based on the VECM can be calculated as Equation 5

$$
\bar{A}_{0} y_{t}=\bar{A}_{1} y_{t-1}+\cdots+\bar{A}_{p} y_{t-p}+\bar{\Xi} D_{t}+\varepsilon_{t},
$$

where $\varepsilon_{t}$ is a $K \times 1$ dimensional vector white noise process with covariance matrix $\Sigma_{\varepsilon}$, which also means structural shocks. $A_{1}, A_{2}, \cdots, A_{p-1}, A_{p}$ are $K \times K$ coefficient matrices. Premultiplying the Equation 5 by $\bar{A}_{0}^{-1}$ can link the reduced form disturbance (forecast errors) $u_{t}$ to the underlying structural shocks $\varepsilon_{t}$.

Based on the prior ordering in the SVECM Cholesky decomposition, the relationship between reduced form residuals and structural shocks are show in Equation 6,

$$
\left[\begin{array}{c}
u_{t}^{\text {UCRY Policy }} \\
u_{t}^{\text {GlobalEPU }} \\
u_{t}^{\text {Vix }} \\
u_{t}^{\text {Bitcoin }} \\
u_{t}^{\text {USFS }} \\
u_{t}^{\text {USEPU }} \\
u_{t}^{\text {Gold }} \\
u_{t}^{\text {UCRYPrice }}
\end{array}\right]=\left[\begin{array}{cccccccc}
S_{11} & 0 & 0 & 0 & 0 & 0 & 0 & 0 \\
S_{21} & S_{22} & 0 & 0 & 0 & 0 & 0 & 0 \\
S_{31} & S_{32} & S_{33} & 0 & 0 & 0 & 0 & 0 \\
S_{41} & S_{42} & S_{43} & S_{44} & 0 & 0 & 0 & 0 \\
S_{51} & S_{52} & S_{53} & S_{54} & S_{55} & 0 & 0 & 0 \\
S_{61} & S_{62} & S_{63} & S_{64} & S_{65} & S_{66} & 0 & 0 \\
S_{71} & S_{72} & S_{73} & S_{74} & S_{75} & S_{76} & S_{77} & 0 \\
S_{81} & S_{82} & S_{83} & S_{84} & S_{85} & S_{86} & S_{87} & S_{88}
\end{array}\right]=\left[\begin{array}{c}
\varepsilon_{t}^{U C R Y P o l i c y} \\
\varepsilon_{t}^{\text {GlobalEPU }} \\
\varepsilon_{t}^{\text {Vix }} \\
\varepsilon_{t}^{\text {Bitcoin }} \\
\varepsilon_{t}^{U S F S} \\
\varepsilon_{t}^{U S E P U} \\
\varepsilon_{t}^{\text {Gold }} \\
\varepsilon_{t}^{\text {UCRYPrice }}
\end{array}\right]
$$

where, $u_{t}$ denotes the reduced form disturbances (forecast errors) at time t. $\varepsilon_{t}$ denotes the structural shocks at time t.

In doing so, we can trace the driving factors of movement in UCRY Policy and UCRY Price. It also enables us to determine UCRY Policy and UCRY Price shocks in our VECM are reflective of uncertainty. The information contained in the historical decomposition could also show the extent to which events are driving shocks to UCRY Policy and UCRY Price.

\section{Results}

Figure 1 shows the weekly values for the derived Policy and Price uncertainty indices based on 726.9 million total news articles collected for the period spanning 2013-2021, and Figure 2 shows the rolling 26-week correlation. We annotated the weekly policy index in Figure 3, highlighting major changes as they map to events in the crypto and related economic spaces.

The historical decomposition of the UCRY Index is shown in Figure 4. The contribution of UCRY Policy shocks to the historical decomposition of the UCRY Index is given in light blue, 
while contribution to the UCRY Price is in orange. These shocks match the expectations of the public to a certain extent. For example, the Brexit vote, Donald Trump winning the 2016 United States presidential election, China banning ICOs, the BTC bubble, DeFi take off and other events have been shown to have positively impacted the UCRY Policy and Price uncertainty Indices. Figure 4 also displays some of the largest hacking attacks of cryptocurrency exchanges, such as attacks on Bitfinex, MintPal, Crispy, and Dao exchanges. These occurred from April 2014 to December 2020, and we have shown that the UCRY Price and UCRY Policy Indices reacted to these events. Fiscal policy adjustments contributed to the small shifts in the UCRY Policy, however, the significance of these events may increase in the future. The decomposition also displayed that UCRY Indices captured uncertainty that could be more distinctively attributed to the major events in cryptocurrencies in comparison to VIX, EPU and Global EPU index. While the price of Bitcoin, the UCRY Policy and the UCRY Price are highly correlated, these indices appear to capture uncertainty beyond Bitcoin prices as shown by the decomposition. Finally, the COVID-19 crisis increased both the UCRY Policy and Price uncertainty Indices, therefore this UCRY Index can be used as an effective measure of uncertainty during the pandemic.

\section{Conclusions}

We have developed a new measure of price and policy uncertainty in cryptocurrency markets. Using 726.9 million news articles from the Lexis Nexis database, we constructed a new Cryptocurrency Uncertainty Index that reflects policy (UCRY Policy) and price uncertainty (UCRY Price) around major cryptocurrencies. This paper provided the historical decomposition of the UCRY Index with major events from 2014 to 2020, such as the COVID-19 crisis, cyberattacks on cryptocurrency exchanges and political elections. Compared to other similar indices it is narrowly range bound, suggesting that while such uncertainty exists, it is not volatile. Nonetheless it does show distinct movements around high profile events in the cryptocurrency space. Our findings suggest that this index can be useful for future research on the uncertainty of cryptocurrency, portfolio

diversification, and contagion effect. Additionally, it can have various practical and policy-based implications for measuring the risk stemming from cryptocurrency markets. 
Table 1: ADF stationarity test

\begin{tabular}{cccc}
\hline Variable & Dickey-Fuller & Lag order & p-value \\
\hline UCRY Policy & -2.101 & 4 & 0.5345 \\
GlobalEPU & -2.7013 & 4 & 0.2881 \\
Vix & -2.4564 & 4 & 0.3886 \\
Bitcoin & -0.43866 & 4 & 0.9824 \\
USFS & -3.252 & 4 & 0.08493 \\
USEPU & -3.0587 & 4 & 0.1414 \\
Gold & -1.1167 & 4 & 0.9146 \\
UCRY Price & -1.6002 & 4 & 0.74 \\
\hline
\end{tabular}

Table 2: The optimal lags

\begin{tabular}{cccc}
\hline $\mathbf{A I C}(\mathbf{n})$ & HQ(n) & SC(n) & FPE(n) \\
\hline 4 & 4 & 4 & 4 \\
\hline
\end{tabular}

Table 3: Johansen test statistic and critical values

\begin{tabular}{ccccc}
\hline & test & 10pct & 5pct & 1pct \\
\hline $\mathbf{r}<=\mathbf{7}$ & 3.83 & 7.52 & 9.24 & 12.97 \\
$\mathbf{r}<=\mathbf{6}$ & 10.58 & 17.85 & 19.96 & 24.60 \\
$\mathbf{r}<=\mathbf{5}$ & 22.99 & 32.00 & 34.91 & 41.07 \\
$\mathbf{r}<=\mathbf{4}$ & 40.32 & 49.65 & 53.12 & 60.16 \\
$\mathbf{r}<=\mathbf{3}$ & 66.04 & 71.86 & 76.07 & 84.45 \\
$\mathbf{r}<=\mathbf{2}$ & 97.06 & 97.18 & 102.14 & 111.01 \\
$\mathbf{r}<=\mathbf{1}$ & 149.28 & 126.58 & 131.70 & 143.09 \\
$\mathbf{r}=\mathbf{0}$ & 215.11 & 159.48 & 165.58 & 177.20 \\
\hline
\end{tabular}

Notes: Johansen trace statistic, lags $=4$ 


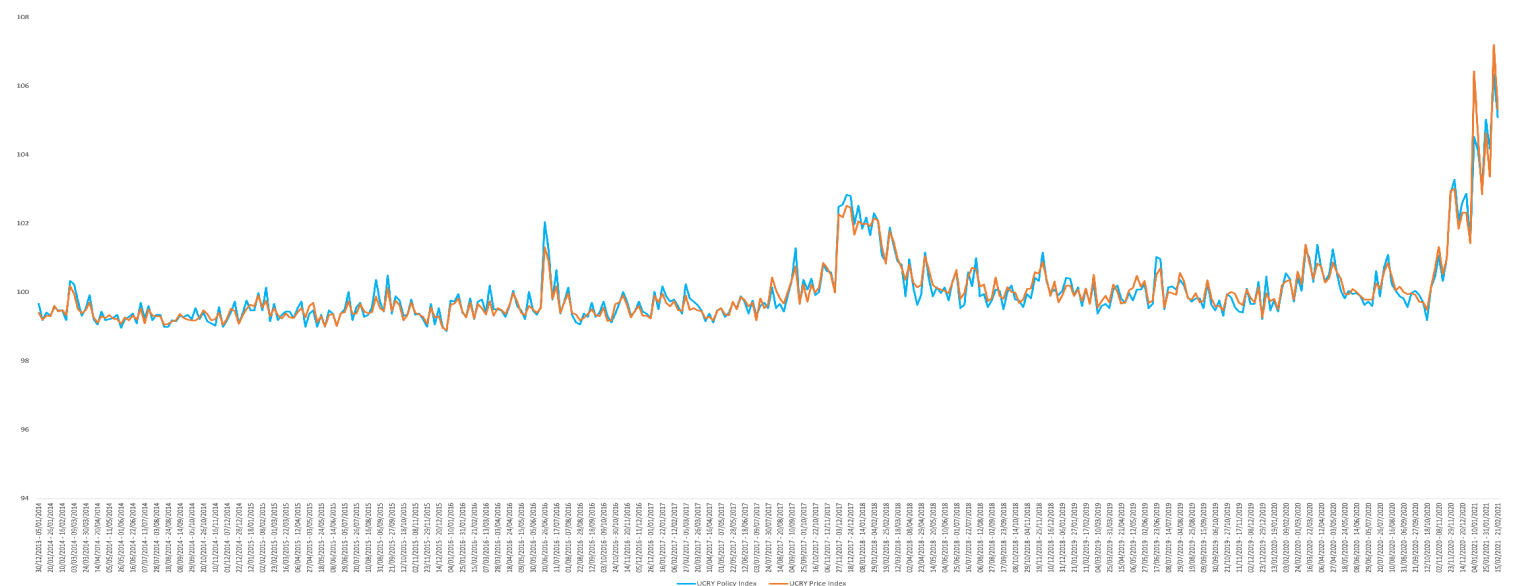

Figure 1: Price and policy uncertainty indices

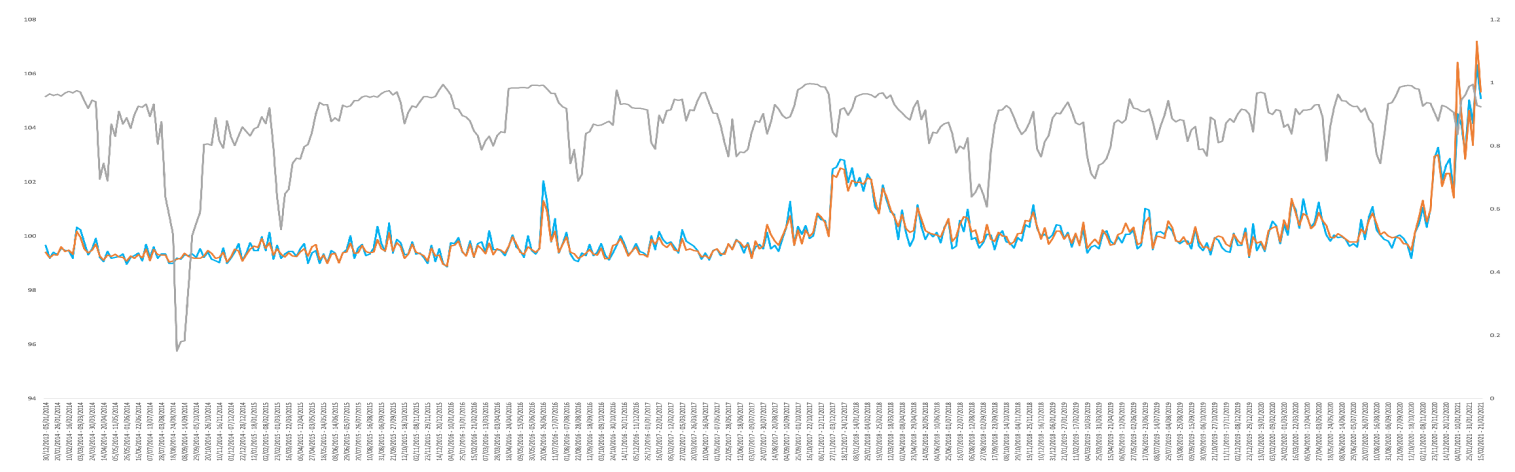

Figure 2: Indices and correlation 


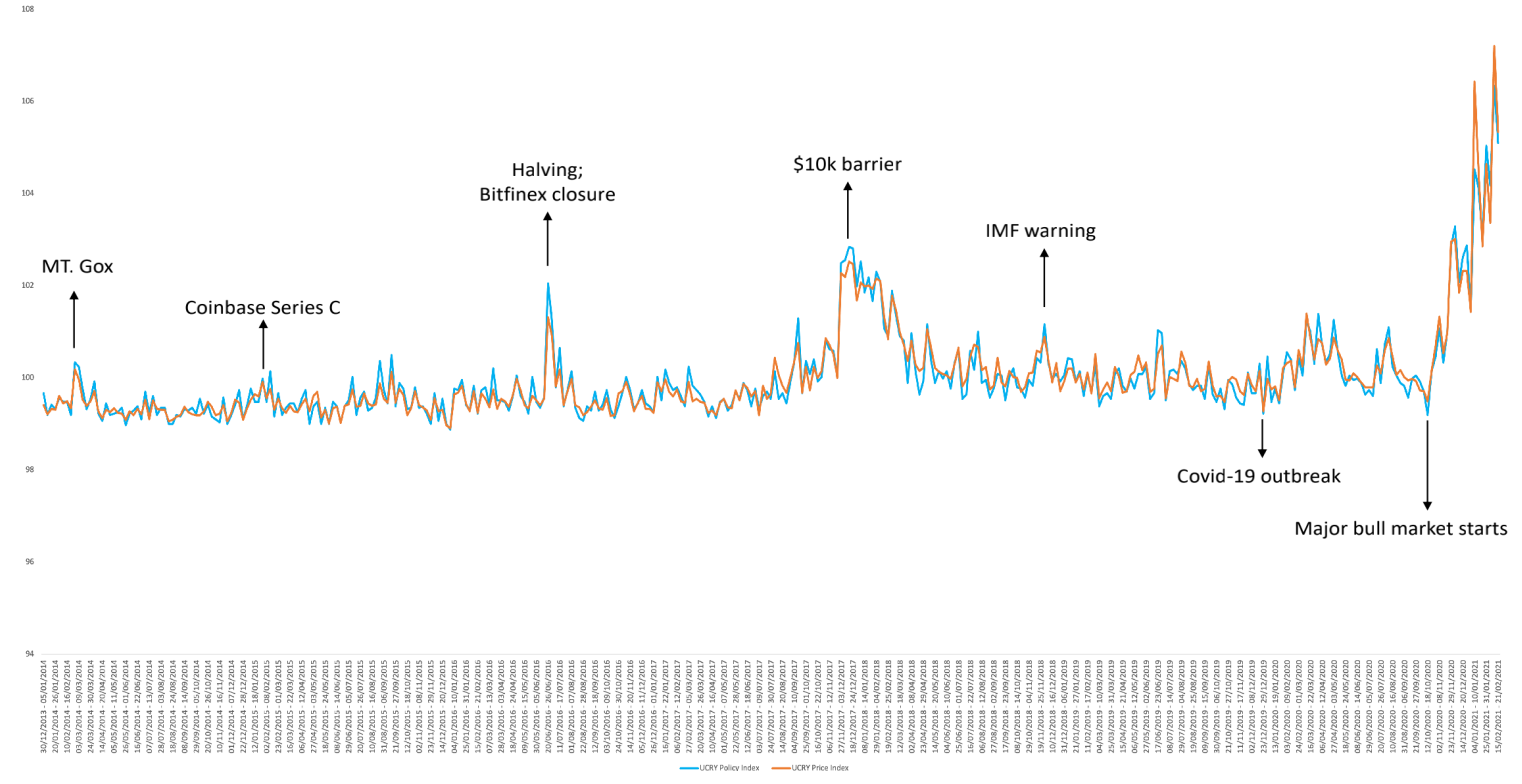

Figure 3: Annotated indices

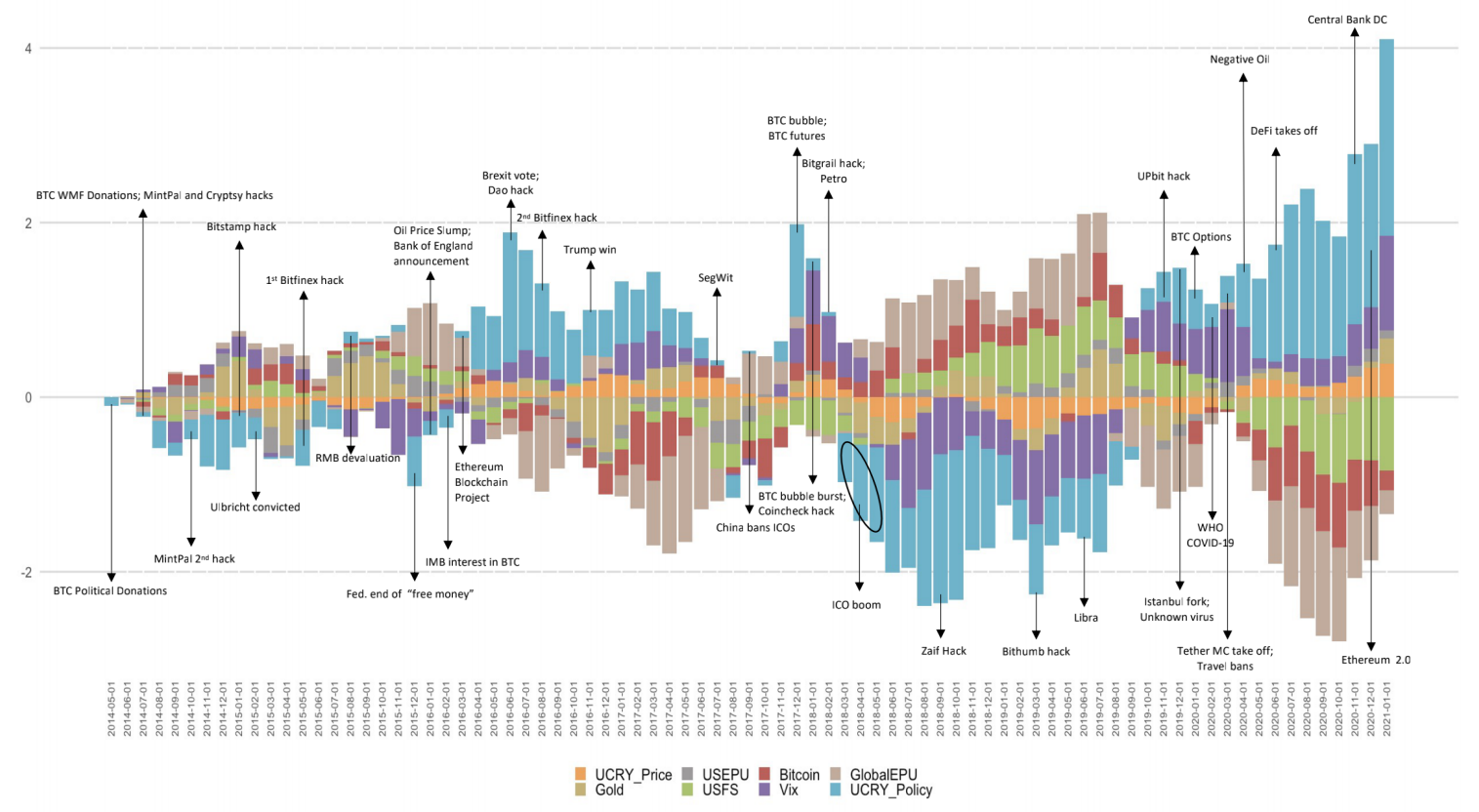

Figure 4: UCRY index historical decomposition with major events 


\section{Bibliography}

\section{References}

Akyildirim, E., S. Corbet, A. Sensoy, and L. Yarovaya (2020). The impact of blockchain related name changes on corporate performance. Journal of Corporate Finance 65.

Akyldirim, E., S. Corbet, B. Lucey, A. Sensoy, and L. Yarovaya (2020). The relationship between implied volatility and cryptocurrency returns. Finance Research Letters 33.

Al Mamun, M., G. Uddin, M. Suleman, and S. Kang (2020). Geopolitical risk, uncertainty and bitcoin investment. Physica A: Statistical Mechanics and its Applications 540.

Aysan, A., E. Demir, G. Gozgor, and C. Lau (2019). Effects of the geopolitical risks on bitcoin returns and volatility. Research in International Business and Finance 47, 511-518.

Baker, S. R., N. Bloom, and S. J. Davis (2016). Measuring economic policy uncertainty. The quarterly journal of economics 131(4), 1593-1636.

Benedetti, H. and E. Nikbakht (2021). Returns and network growth of digital tokens after cross-listings. Journal of Corporate Finance 66 .

Conlon, F., C. Kim, H. Kim, and W. Kim (2020). The effect of political and economic uncertainty on the cryptocurrency market. Finance Research Letters In Press.

Corbet, S., C. Larkin, B. M. Lucey, A. Meegan, and L. Yarovaya (2020a). Cryptocurrency reaction to fomc announcements: Evidence of heterogeneity based on blockchain stack position. Journal of Financial Stability 46.

Corbet, S., C. Larkin, B. M. Lucey, A. Meegan, and L. Yarovaya (2020b). The impact of macroeconomic news on bitcoin returns. The European Journal of Finance 26, 1396-1416.

Corbet, S., A. Meegan, C. Larkin, B. Lucey, and L. Yarovaya (2018). Exploring the dynamic relationships between cryptocurrencies and other financial assets. Economics Letters 165, 28-34.

Davis, S. (2016). An index of global economic policy uncertainty. NBER working paper No. 22740.

Demir, E., G. Gozgor, C. K. M. Lau, and S. A. Vigne (2018). Does economic policy uncertainty predict the bitcoin returns? an empirical investigation. Finance Research Letters 26, 145-149. https://doi.org/10.1016/j.frl.2018.01.005.

Fang, T., Z. Su, and L. Yin (2020). Economic fundamentals or investor perceptions? the role of uncertainty in predicting long-term cryptocurrency volatility. International Review of Financial Analysis 71.

Gozgor, G., A. Tiwari, E. Demir, and S. Akron (2020). The relationship between bitcoin returns and trade policy uncertainty. Finance Research Letters 29, 75-82.

Huang, Y. and P. Luk (2020). Measuring economic policy uncertainty in china. China Economic Review 59

Lütkepohl, H. (2005). New introduction to multiple time series analysis. Springer Science \& Business Media.

Manela, A. and A. Moreira (2017). News implied volatility and disaster concerns. Journal of Financial Economics 123, 137-162. 
Phillips, R. C. and D. Gorse (2017). Predicting cryptocurrency price bubbles using social media data and epidemic modelling. In 2017 IEEE symposium series on computational intelligence (SSCI), pp. 1-7. IEEE.

Rice, J. (2020). Economic policy uncertainty in small open economies: a case study in ireland. Research Technical Papers 01/RT/20, Central Bank of Ireland.

Subramaniam, S. and M. Chakraborty (2020). Investor attention and cryptocurrency returns: Evidence from quantile causality approach. Journal of Behavioral Finance 21(1), 103-115.

Trimborn, S. and W. Härdle (2018). Crix an index for cryptocurrencies. Journal of Empirical Finance 49, 107-122.

Walther, T., T. Klein, and E. Bouri (2019). Exogenous drivers of bitcoin and cryptocurrency volatility - a mixed data sampling approach to forecasting. Journal of International Financial Markets, Institutions and Money 63, 101133. https://doi.org/10.1016/j.intfin.2019.101133.

Wu, S., M. Tong, Z. Yang, and A. Derbali (2019). Does gold or bitcoin hedge economic policy uncertainty? Finance Research Letters 31, 171-178.

Yarovaya, L. and D. Zięba (2020). Intraday volume-return nexus in cryptocurrency markets: A novel evidence from cryptocurrency classification. Available at SSRN: https://ssrn.com/abstract=3711667. 


\section{Appendix}

Appendix A - Figure

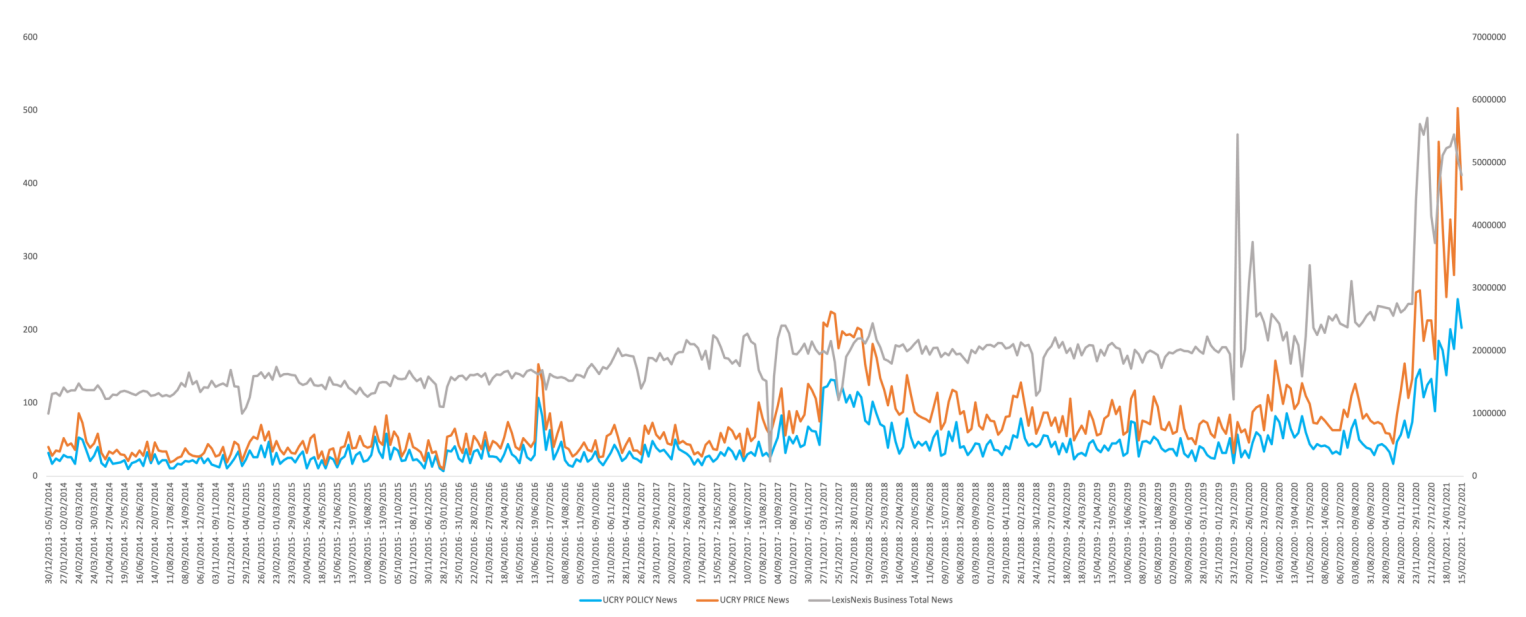

Figure 5: News trend 
Appendix B - Tables

Table 4: Descriptive statistics

\begin{tabular}{cccccccccc}
\hline Variables & Count & Minimum & Maximum & Mean & Median & Variance & Stdev & Skewness & Kurtosis \\
\hline UCRY Policy & 85 & 99.1009 & 104.3415 & 99.9755 & 99.759 & 0.714393 & 0.8452 & 2.5765 & 8.4451 \\
GlobalEPU & 85 & 86.17 & 429.43 & 192.9751 & 168.95 & 6261.7856 & 79.1314 & 0.7316 & -0.2937 \\
Vix & 85 & 9.5100 & 53.54 & 17.4523 & 15.08 & 55.420802 & 7.4445 & 2.2090 & 6.1422 \\
Bitcoin & 85 & $2.296700 \mathrm{e}+02$ & $3.464109 \mathrm{e}+04$ & $4.997788 \mathrm{e}+03$ & $2.886710 \mathrm{e}+03$ & $3.726869 \mathrm{e}+07$ & $6.104809 \mathrm{e}+03$ & $2.225124 \mathrm{e}+00$ & $7.073033 \mathrm{e}+00$ \\
USFS & 85 & 100.05 & 102.13 & 100.5342 & 100.52 & 0.1025 & 0.320221 & 1.7517 & 6.5731 \\
USEPU & 85 & 29.32 & 557.19 & 119.4641 & 94.57 & 9742.6910 & 98.7050 & 2.4928 & 6.9873 \\
Gold & 85 & 1061.10 & 1975.86 & 1344.3094 & 1283.53 & 45934.2393 & 214.3228 & 1.48994 & 1.4250 \\
UCRY Price & 85 & 99.091 & 104.8057 & 99.9766 & 99.7759 & 0.737670 & 0.8589 & 2.7865 & 10.8790 \\
\hline
\end{tabular}

Table 5: Pearson correlation

\begin{tabular}{ccccccccc}
\hline & UCRY Policy & GlobalEPU & Vix & Bitcoin & USFS & USEPU & Gold & UCRY Price \\
UCRY Policy & 1.0000 & & & & & & & \\
GlobalEPU & 0.3886 & 1.0000 & & & & & & \\
Vix & 0.3107 & 0.5257 & 1.0000 & & & & & \\
Bitcoin & 0.8299 & 0.5910 & 0.3879 & 1.0000 & & & & \\
USFS & 0.0203 & -0.0464 & 0.1167 & -0.0564 & 1.0000 & & & \\
USEPU & 0.3287 & 0.7398 & 0.6682 & 0.4282 & 0.3192 & 1.0000 & & \\
Gold & 0.4691 & 0.7417 & 0.5717 & 0.7441 & -0.0237 & 0.6387 & 1.0000 & \\
UCRY Price & 0.9894 & 0.3994 & 0.3230 & 0.8589 & 0.0156 & 0.3198 & 0.4902 & 1.0000 \\
\hline
\end{tabular}




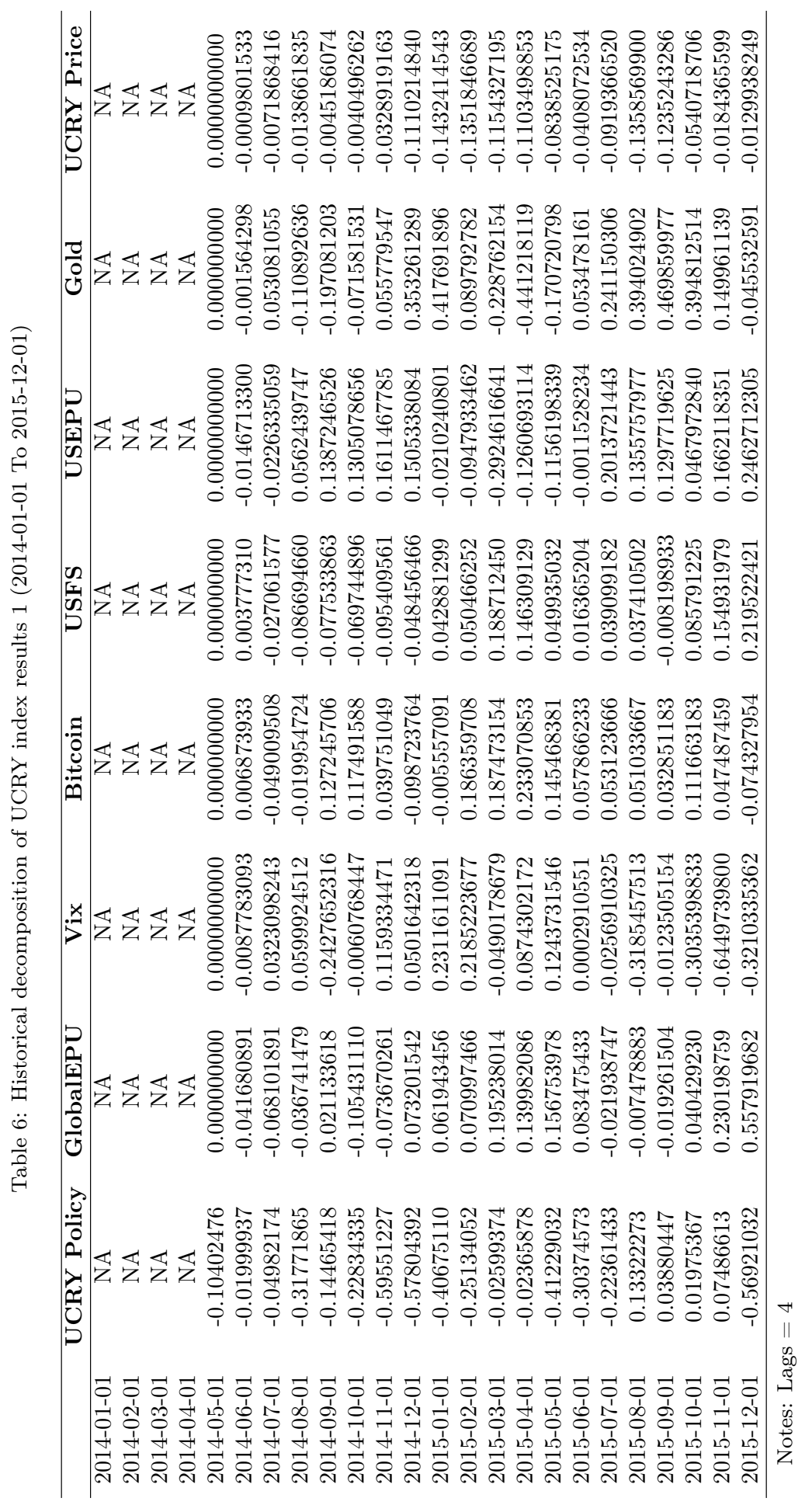




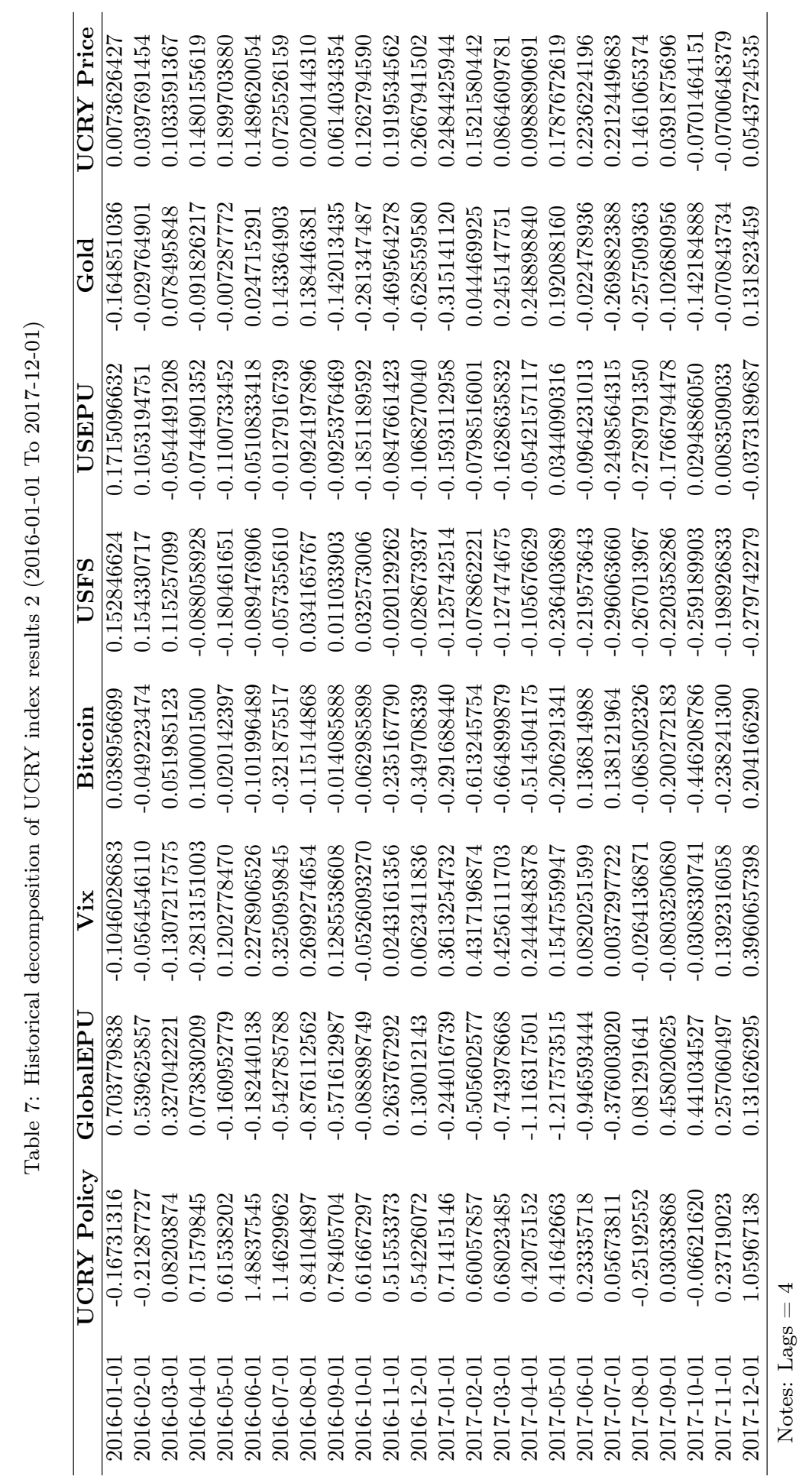




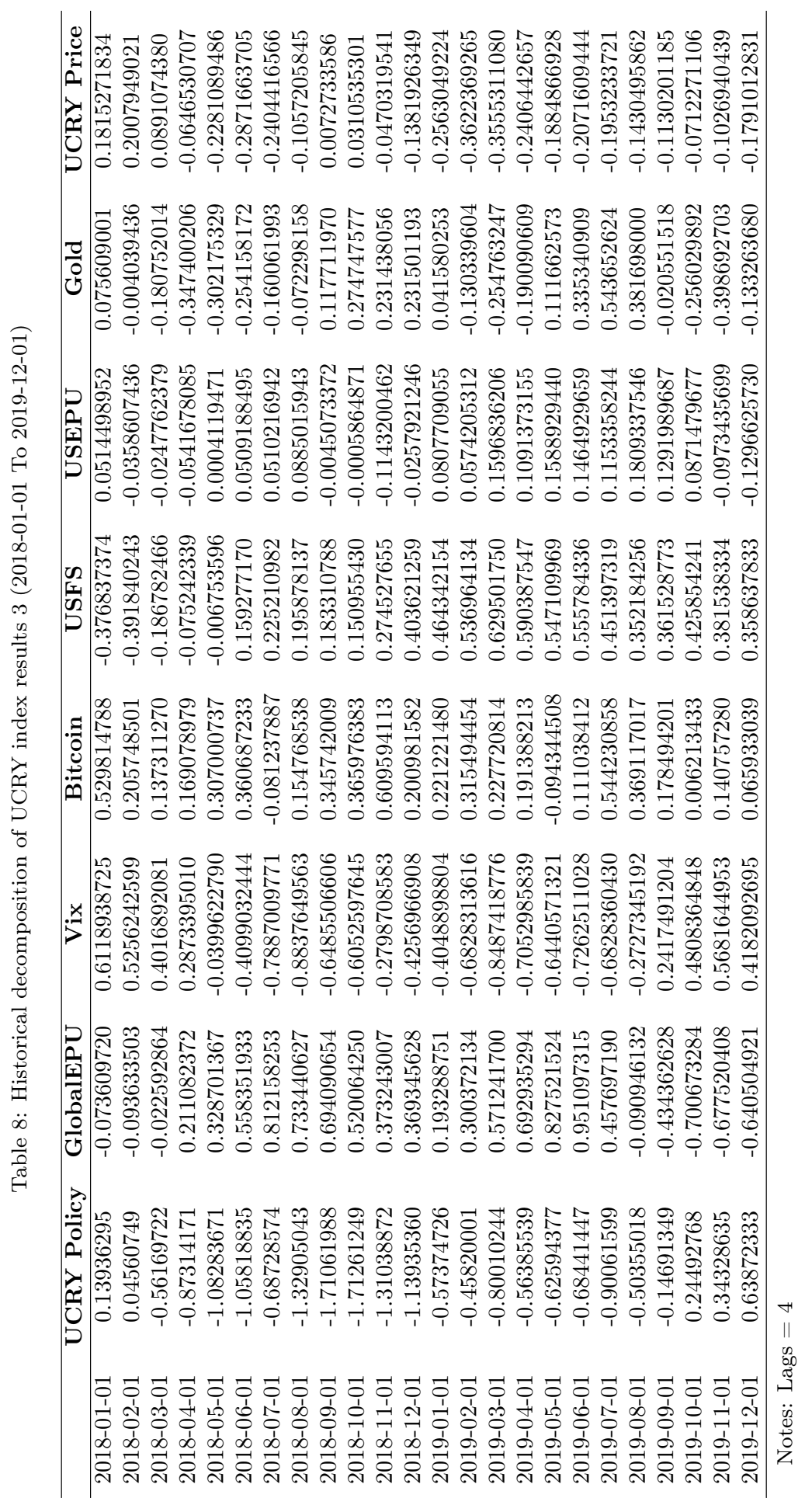




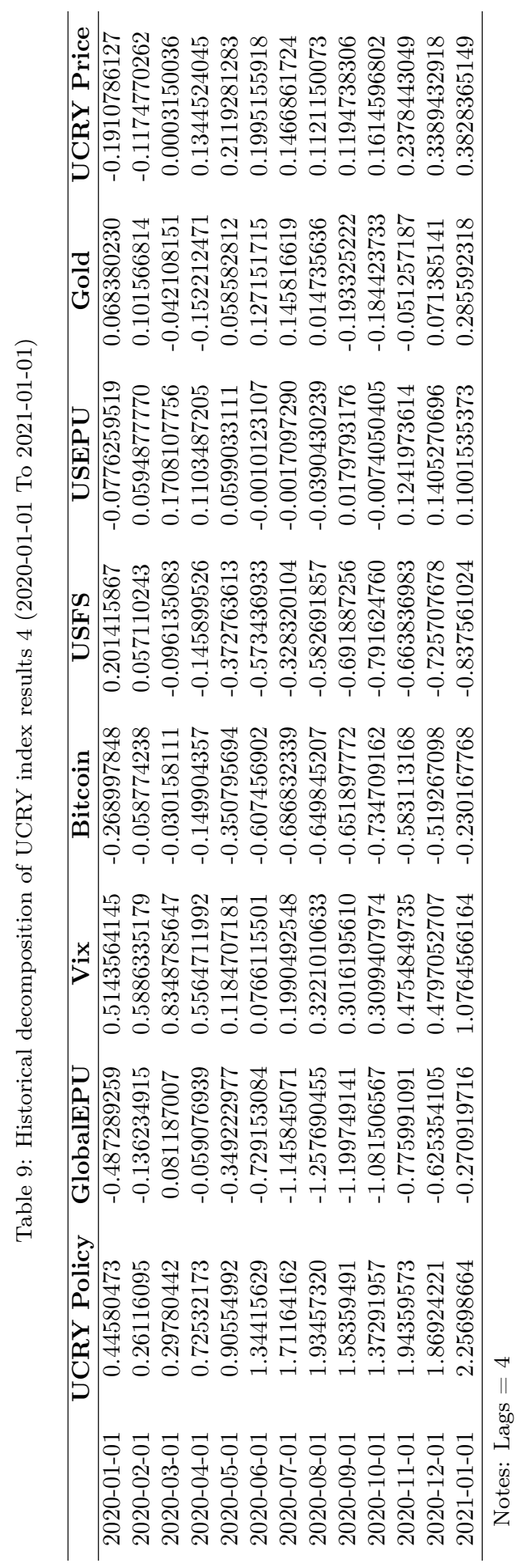

